Shimura, G.

Osaka J. Math.

1 (1964), 1-14.

\title{
ON PURELY TRANSCENDENTAL FIELDS OF AUTOMORPHIC FUNCTIONS OF SEVERAL VARIABLES
}

\author{
GoRo SHIMURA
}

(Received December 2, 1963)

The purpose of this paper is to give some examples of arithmetically defined discontinuous groups $\Gamma$ operating on a complex ball

$$
H^{r}=\left\{\left.\left(z_{1}, \cdots, z_{r}\right) \in C^{r}|| z_{1}\right|^{2}+\cdots+\left|z_{r}\right|^{2}<1\right\}
$$

such that the field of all automorphic functions ${ }^{1)}$ on $H^{r}$ with respect to $\Gamma$ is a purely transcendental extension of $\boldsymbol{C}$ of dimension $r$. To get such a $\Gamma$, we consider the field $K=\boldsymbol{Q}(\zeta)$ generated by a primitive $m$-th root of unity $\zeta$, and take a hermitian matrix $S$ of size $r+1$ with entries in $K$ such that $S$ itself has exactly $r$ positive and one negative characteristic roots while all the other conjugates of $S$ over $\boldsymbol{Q}$ are definite. Let $U_{0}(S)$ be the group of all complex matrices $X$ such that ${ }^{t} \bar{X} S X=S$. Let $\Gamma$ be the subgroup of $U_{0}(S)$ consisting of the matrices whose entries are algebraic integers in $K$. Since $H^{r}$ is isomorphic to the quotient space of $U_{0}(S)$ with respect to a maximal compact subgroup, $\Gamma$ operates naturally on $H^{r}$. In our examples, the automorphic functions with respect to $\Gamma$ give moduli of algebraic curves $Y: y^{m}=p(x)$, where $p(x)$ is a polynomial in $\boldsymbol{C}[x]$. Then the following table describes our examples.

\begin{tabular}{|c|c|c|c|c|l|}
\hline & $K$ & $r$ & \multicolumn{1}{|c}{$S$} & \multicolumn{1}{|c}{$H^{r} / \Gamma$} & \multicolumn{1}{|c}{$Y$} \\
\hline$(1)$ & $\boldsymbol{Q}\left(1^{1 / 3}\right)$ & 2 & $\operatorname{diag}[1,1,-1]$ & non-compact & $y^{3}=p_{4}(x)$ \\
\hline$(2)$ & $\boldsymbol{Q}\left(1^{1 / 3}\right)$ & 3 & $\operatorname{diag}[1,1,1,-1]$ & non-compact & $y^{3}=p_{6}(x)$ \\
\hline$(3)$ & $\boldsymbol{Q}\left(1^{1 / 4}\right)$ & 2 & $\operatorname{diag}[1,1,-1]$ & non-compact & $y^{4}=p_{2}(x) p_{3}(x)^{2}$ \\
\hline$(4)$ & $\boldsymbol{Q}\left(1^{1 / 5}\right)$ & 1 & $\operatorname{diag}[1,(1-\sqrt{5}) / 2]$ & compact & $y^{5}=p_{3}(x)$ \\
\hline$(5)$ & $\boldsymbol{Q}\left(1^{1 / 5}\right)$ & 2 & $\operatorname{diag}[1,1,(1-\sqrt{5}) / 2]$ & compact & $y^{5}=p_{5}(x)$ \\
\hline$(6)$ & $\boldsymbol{Q}\left(1^{1 / 7}\right)$ & 1 & $\operatorname{diag}\left[1,-\frac{\sin (3 \pi / 7)}{\sin (2 \pi / 7)}\right]$ & compact & $y^{7}=p_{3}(x)$ \\
\hline
\end{tabular}

1) By an automorphic function, we always mean a meromorphic function which is invariant under the operation of the group in question. 
Here $1^{1 / m}$ denotes a primitive $m$-th root of unity, diag $\left[a_{1}, \cdots, a_{s}\right]$ the diagonal matrix with diagonal elements $a_{1}, \cdots, a_{s}$, and $p_{n}(x)$ a polynomial of degree $n$ and without multiple root.

Theorem. In these six cases, the field of all automorphic functions on $H^{r}$ with respect to $\Gamma$ is a purely transcendental extension of $\boldsymbol{C}$ of dimension $r$.

It would be worth while mentioning the following point. There was previously no known example of a discontinuous group $\Gamma$ operating on a bounded symmetric domain $D$ of dimension $>1$ such that $D / \Gamma$ is compact and the field of all automorphic functions on $D$ with respect to $\Gamma$ is purely transcendental over $\boldsymbol{C}$. The case (5) gives actually such a discontinuous group.

Picard [3] investigated the curve $y^{3}=p_{4}(x)$ and observed that moduli of such curves give automorphic functions on $H^{2}$. But it seems that he did not determine the whole field of automorphic functions.

To prove our theorem, we consider the canonically polarized jacobian variety $J$ of the algebraic curve $Y$. It turns out that $J$ belongs to an analytic family $\Sigma$ treated in our previous paper [6]. In the above cases, if $Y$ is a generic curve of the given type, $J$ is a generic member of $\Sigma$. Now the moduli of $Y$ are, roughly speaking, the same as the moduli of $J$. Then the main theorem of [6] shows that the moduli of $Y$ are given by the automorphic functions with respect to a certain discontinuous group $\Gamma$. In order to determine the explicit form of $\Gamma$, we need some analysis of lattices in a vector space over $K$ with a hermitian form, which was one of the subjects investigated in [7]. In the Appendix, we give a supplement to it.

In the present paper, we treated the moduli of $Y$ only at generic points. It would be interesting to study the moduli of $Y$ in more detail, for example, from the view-point of Igusa [2], who investigated the moduli of algebraic curves of genus two.

1. First we recall some results of [6]. Let $F$ be a totally real algebraic number field of degree $g$, and $K$ a totally imaginary quadratic extension of $F$. We denote by $\rho$ the complex conjugation. Let $\Phi$ be a representation of $K$ by complex matrices of size $h$. We say that a triplet $\mathbb{P}=(A, \mathcal{C}, \theta)$ is a polarized abelian variety of type $\{K, \Phi, \rho\}$ if the following conditions are satisfied.

(i) $A$ is an abelian variety of dimension $h$, defined over $\boldsymbol{C}$.

(ii) $\theta$ is an isomorphism of $K$ into $\operatorname{End}_{Q}(A)$; and the representation of $\theta(x)$ for $x \in K$ by an analytic coordinate system of $A$ is equivalent to $\Phi$. 
(iii) $\mathcal{C}$ is a polarization of $A$; and the involution of $\operatorname{End}_{\boldsymbol{Q}}(A)$ determined by $\mathcal{C}$ coincides with $\theta(x) \rightarrow \theta\left(x^{\rho}\right)$ on $\theta(K)$.

Let $\sigma_{1}, \cdots, \sigma_{g}, \sigma_{1} \rho, \cdots, \sigma_{g} \rho$ be all the isomorphisms of $K$ into $\boldsymbol{C}$, and let $r_{\nu}$ (resp. $s_{\nu}$ ) be the multiplicity of $\sigma_{\nu}$ (resp. $\sigma_{\nu} \rho$ ) in $\Phi$. In order to insure the existence of $\mathcal{P}$ of type $\{K, \Phi, \rho\}$, the following relation should be satisfied $[6,2.1]$ :

$$
h=g\left(r_{\nu}+s_{\nu}\right) \quad(1 \leqq \nu \leqq g) .
$$

Hereafter we assume (1.1) and put $u=h / g$.

Let $P=(A, \mathcal{C}, \theta)$ be of type $\{K, \Phi, \rho\}$. Take a complex torus $\boldsymbol{C}^{h} / D$ isomorphic to $A$, where $D$ is a lattice in $C^{h}$. We may choose the coordinate system of $\boldsymbol{C}^{h}$ so that $\theta(a)$ is represented by the matrix $\Phi(a)$ on $\boldsymbol{C}^{h}$ for every $a \in K$. Let $K^{u}$ be the vector space of all $u$-dimensional row vectors with components in $K$. Then we find $u$ vectors $x_{1}, \cdots, x_{u}$ in $\boldsymbol{C}^{h}$ such that $\boldsymbol{Q} D=\sum_{i=1}^{u} \Phi(K) x_{i}$. For every $a=\left(a_{1}, \cdots, a_{u}\right)$ in $K^{u}$, put $x(a)$ $=\sum_{i=1}^{u} \Phi\left(a_{i}\right) x_{i}$. Then the mapping $a \rightarrow x(a)$ is an isomorphism of $K^{u}$ onto $\boldsymbol{Q} D$. Let $L$ be the inverse image of $D$ by this mapping.

Let $E(x, y)$ be a Riemann form on $C^{h} / D$ corresponding to a basic polar divisor in $\mathcal{C}$. Then there exists an anti-hermitian form $T(a, b)$ on $K^{u}$ such that

$$
E(x(a), \neq x(b))=\operatorname{Tr}_{K / Q}(T(a, b)) \quad\left((a, b) \in K^{u} \times K^{u}\right) .
$$

The structure $\left\{K^{u}, T, L\right\}$ is uniquely determined by $\odot$ up to isomorphism. We say that $P$ is of type $\{K, \Phi, \rho ; T, L\}$. We note that $T$ can not be arbitrary ; it must satisfy the following condition $[6$, p. 160, (25)]:

(1.3) The hermitian matrix $\sqrt{-1} T^{\sigma_{\nu}}$ has the same signature as $\left[\begin{array}{cc}-1_{\gamma_{\nu}} & 0 \\ 0 & 1_{s_{v}}\end{array}\right]$ for every $\nu$, where $1_{r}$ denotes the identity matrix of degree $r$.

Let $H_{\nu}$ be the space of all complex matrices $z$ with $r_{\nu}$ rows and $s_{\nu}$ columns such that $1-{ }^{t} \bar{z} z$ is positive hermitian, and let

$$
H=H_{1} \times \cdots \times H_{g} .
$$

Then we get an analytic family $\Sigma(K, \Phi, \rho ; T, L)=\left\{\mathcal{P}_{z} \mid z \in H\right\}$ of polarized abelian varieties $\rho_{z}$ of type $\{K, \Phi, \rho ; T, L\}$ parametrized by the point $z$ on $H$. Every $\odot$ of type $\{K, \Phi, \rho ; T, L\}$ is isomorphic to a member of $\Sigma(K, \Phi, \rho ; T, L)$.

Now we let every element of $M_{u}(K)$ operate on $K^{u}$ on the right, and define a group $\Gamma(T, L)$ by

$$
\Gamma(T, L)=\left\{X \in G L_{u}(K) \mid T(a X, b X)=T(a, b), L X=L\right\} .
$$


Then $\Gamma(T, L)$ gives a properly discontinuous group of transformations on $H[6,2.7]$. In [6, Th. 3], we get meromorphic functions $f_{1}, \cdots, f_{\kappa}$ on $H$ and an analytic subset $W$ of $H$ of codimension one, such that $\boldsymbol{Q}\left(f_{1}(z), \cdots\right.$, $\left.f_{k}(z)\right)$ is the field of moduli of $P_{z}$ for every $z \in H-W$. As remarked in [6, p. 172], if $H / \Gamma(T, L)$ is compact, $C\left(f_{1}, \cdots, f_{\kappa}\right)$ is the field of all automorphic functions on $H$ with respect to $\Gamma(T, L)$. Even if $H / \Gamma(T, L)$ is not compact, the last statement is true in view of [6, Th.4] and a recent result of Baily and Borel on the compactification of $H / \Gamma(T, L)$.

Proposition 1. Let $P$ be of type $\{K, \Phi, \rho ; T, L\}$ and $k_{0}$ the field of moduli of $\mathcal{P}$. If $\operatorname{dim}_{\boldsymbol{Q}} k_{0}=\sum_{v=1}^{g} r_{\nu} s_{\nu}$, then $\boldsymbol{Q}\left(f_{1}, \cdots, f_{\kappa}\right)$ is isomorphic to $k_{0}$.

This follows from [6, Theorem 4, (iii)] and [5, Prop. 3.5 and p. 305, Remark].

2. Let $m$ and $n$ be positive integers. Let $Y$ be an algebraic curve defined by $y^{m}=p(x)$, where $p(x)$ is a polynomial in $C[x]$, of degree $n$ and without multiple root. If $d$ is the greatest common divisor of $m$ and $n$, the genus $h$ of $Y$ is given by

$$
h=\frac{1}{2}[(m-1)(n-1)-(d-1)] .
$$

The vector space of differential forms of the first kind on $Y$ is spanned by the $x^{a} d x / y^{b}$ with integers $a$ and $b$ satisfying $0 \leqq a<n, 0<b<m$, $b n-a m-m-d \geqq 0$.

If $m$ divides $n+1$, take a complex number $c$ so that $p(c) \neq 0$, and put $v=1 /(x-c), n+1=m e$. Then we get $\left(v^{e} y\right)^{m}=v \cdot v^{n} p\left(v^{-1}+c\right)$. This shows that $Y$ is birationally equivalent to the curve $y^{m}=q(x)$ with a polynomial $q(x)$ in $C[x]$ of degree $n+1$ and without multiple root.

Hereafter we assume that $m$ does not divide $n+1, h>1$, and $m$ is an odd prime number. Let $J$ be the jacobian variety of $Y$, and $\rho$ a canonical mapping of $Y$ into $J$. We fix a primitive $m$-th root of unity $\zeta$. Let $\zeta_{0}$ be the birational correspondence of $Y$ with itself given by $(x, y) \rightarrow(x, \zeta y)$. Denote by $\theta(\zeta)$ the automorphism of $J$ corresponding to $\zeta_{0}$. We see that $\zeta \rightarrow \theta(\zeta)$ can be extended naturally to an isomorphim $\theta$ of $\boldsymbol{Q}(\zeta)$ into $\operatorname{End}_{Q}(J)$. Let $C$ be the canonical polarization of $J$, and $\rho$ the automorphism of $\boldsymbol{Q}(\zeta)$ such that $\zeta^{\rho}=\zeta^{-1}$. The involution of $\operatorname{End}_{Q}(J)$ determined by $\mathcal{C}$ gives the automorphism $\theta(a) \rightarrow \theta\left(a^{p}\right)$ on $\theta(\boldsymbol{Q}(\zeta))$. In this way we get a polarized abelian variety of type $\{\boldsymbol{Q}(\zeta), \Phi, \rho\}$ in the sense of $\S 1$, for a certain representation $\Phi$ of degree $h$. In view of the explicit form of differential forms of the first kind given above, we see that, for every integer $b$ such that $0<b<m$, the matrix $\left.\Phi_{(}^{\prime} \zeta\right)$ has $\zeta^{-b}$ as 
a characteristic root with multiplicity $[(b n-d) / m]$, where $[\alpha]$ denotes the largest non-negative integer $\leqq \alpha$.

3. Let $Y^{*}$ be another curve defined by $y^{m}=p^{*}(x)$ for a polynomial $p^{*}(x)$ in $C[x]$ of degree $n$ and without multiple root. From $Y^{*}$, we obtain a polarized abelian variety $\rho^{*}=\left(J^{*}, \mathcal{C}^{*}, \theta^{*}\right)$ of type $\{\boldsymbol{Q}(\zeta), \Phi, \rho\}$ in the same way as above; we note that the representation $\Phi$ is the same for fixed $m$ and $n$. Let $\zeta_{0}^{*}$ be the birational correspondence of $Y^{*}$ with itself given by $(x, y) \rightarrow(x, \zeta y)$.

Proposition 2. $\odot$ is isomorphic to $\mathcal{P}^{*}$ if and only if there exists a birational mapping $\lambda$ of $Y$ to $Y^{*}$ such that $\lambda \zeta_{0}=\zeta_{0}^{*} \lambda$.

The 'if' part is obvious. Let $\mathcal{P}^{*}$ be a canonical mapping of $Y^{*}$ to $J^{*}$. Suppose that there exists an isomorphism $\mu$ of $\mathcal{P}$ to $\mathcal{P}^{*}$. By Torelli's theorem, there exists a birational mapping $\lambda$ of $Y$ to $Y^{*}$ such that $\mathcal{P}^{*} \lambda= \pm \mu \cdot \mathcal{P}+a$, where $a$ is a point of $J^{*}$. Since $\mu \theta(\zeta)=\theta^{*}(\zeta) \mu$, we see easily that $\lambda^{-1} \zeta_{0}^{*} \lambda$ and $\zeta_{0}$ correspond to the same automorphism $\theta(\zeta)$ of $J$. By our assumption $h>1$, we must have $\lambda^{-1} \zeta_{0}^{*} \lambda=\zeta_{0}$. Our proposition is thereby proved.

Proposition 3. Let $k_{0}$ be the composite of $\boldsymbol{Q}(\zeta)$ and the field of moduli of $P$. Then $k_{0}$ is the subfield of $\boldsymbol{C}$ which is uniquely determined by the following properties.

$\left(M_{1}\right)$ If $k$ is a field of definition for $Y$ and $\zeta_{0}$, then $k \supset k_{0}$. If furthermore $\sigma$ is an isomorphism of $k$ into $\boldsymbol{C}$, over $\boldsymbol{Q}(\zeta)$, then $\sigma$ is the identity mapping on $k_{0}$ if and only if there exists a birational mapping $\lambda$ of $Y$ to $Y^{\sigma}$ such that $\lambda \zeta_{0}=\zeta_{0}^{\sigma} \lambda$.

$\left(M_{2}\right) \quad k_{0} \supset \boldsymbol{Q}(\zeta)$

This follows immediately from Prop. 2 and the definition of the field of moduli of $P[4$, p. 110].

Proposition 4. Let $\lambda$ be a birational mapping of $Y$ to $Y^{*}$ such that $\lambda \zeta_{0}=\zeta_{0}^{*} \lambda$, and let $\lambda(x, y)=(u, v)$. Then $u$, $v$ are rational expressions of $x, y$ of the following form.

(I) If $m$ divides $n, u=(a x+b) /(c x+d), v=e y /(c x+d)^{n / m}$.

(II) If $m$ does not divide $n, u=a x+b, v=e y$.

Here $a, b, c, d, e$ are complex numbers.

Let $u=\sum_{i=0}^{m-1} r_{i}(x) y^{i}, v=\sum_{i=0}^{m-1} s_{i}(x) y^{i}$ with $r_{i}(x)$ and $s_{i}(x)$ in $C(x)$. Since $\lambda \zeta_{0}=\zeta_{0}^{*} \lambda$, we have $\sum_{i=0}^{n-1} r_{i}(x) \zeta^{i} y^{i}=\sum_{i=0}^{m-1} r_{i}(x) y^{i}, \sum_{i=0}^{n-1} s_{i}(x) \zeta^{i} y^{i}$ 
$=\zeta \sum_{i=0}^{m-1} s_{i}(x) y^{i}$, so that $u=r_{0}(x), v=s_{1}(x) y$. Since $\lambda$ is one-to-one, $r_{0}$ must be linear fractional : $r_{0}(x)=(a x+b) /(c x+d)$. Write $s_{1}(x)$ as $s_{1}(x)$ $=s(x) / t(x)$ with polynomials $s(x)$ and $t(x)$ which are relatively prime. Then we get

$$
s(x)^{m}(c x+d)^{n} p(x)=t(x)^{m}(c x+d)^{n} p^{*}((a x+b) /(c x+d)) .
$$

We see that $(c x+d)^{n} p^{*}((a x+b) /(c x+d))$ is a polynomial in $x$ of degree $n$ or $n-1$, without multiple root. It follows that $s(x)$ is a constant. Recall that we excluded the case $m \mid n+1$. Then we get easily our assertions.

Suppose that $m$ divides $n$. We see easily that the transformation of (I) of Prop. 4 always gives a birational mapping of $Y$ to another curve $v^{m}=p^{*}(u)$ with a polynomial $p^{*}(u)$ of degree $n$ or $n-1$, without multiple root. If $a / c$ is not a root of $p(x), p^{*}(u)$ is of degree $n$.

If $m$ does not divide $n$, it is clear that the transformation of (II) of Prop. 4 gives a birational mapping of $Y$ to a curve of the same type.

4. Let $q$ be a polynomial in $C[x]$ of degree $\leqq n$, other than 0 , and let $q(x)=\sum_{i=0}^{n} q_{i} x^{i}$. Let $P^{n}$ be the projective space of dimension $n$. Denote by $[q]$ the point $\left(q_{0}, \cdots, q_{n}\right)$ in $P^{n}$. Let $\alpha=\left(\begin{array}{ll}a & b \\ c & d\end{array}\right)$ be a generic point of $G L_{2}(\boldsymbol{C})$ over $\boldsymbol{Q}\left(q_{0}, \cdots, q_{n}\right)$ and let $q^{\alpha}$ be the polynomial determined by

$$
q^{\alpha}(x)=(c x+d)^{n} q((a x+b) /(c x+d)) .
$$

We denote by $W(q)$ the locus of $\left[q^{\alpha}\right]$ over $\boldsymbol{Q}\left(q_{0}, \cdots, q_{n}\right)$. It can be easily seen that $W(q)$ is a variety determined only by $q$, and independent of the choice of $\alpha$. By Prop. 4 and by a standard argument, we get

Proposition 5. Suppose that $m$ divides $n$. Let $Y$ and $Y^{*}$ be as in $\S \S 2,3$. Then $W(p)=W\left(p^{*}\right)$ if and only if there exists a birational mapping $\lambda$ of $Y$ to $Y^{*}$ such that $\lambda \zeta_{0}=\zeta_{0}^{*} \lambda$.

From this and Prop. 3, we obtain

Proposition 6. Suppose that $m$ divides $n$. Let $c$ be the Chow point of $W(p)$. Then $\boldsymbol{Q}(\zeta, c)$ is the field $k_{0}$ of Prop. 3.

Let $p(x)=\sum_{i=0}^{n} p_{i} x^{i}$. If $p_{0}, \cdots, p_{n}$ are algebraically independent over $\boldsymbol{Q}$, we see easily that $\boldsymbol{Q}(c)$ is the field of all quotients of homogeneous invariants, in the classical sense, of the binary form $\sum_{i=0}^{n} p_{i} x^{i} y^{n-i}$. In particular, if $n=5$, it is known that every invariant is a polynomial of certain invariants $A, B, C, R$ of degree $4,8,12,18$; and $R^{2}$ is a poly- 
nomial of $A, B, C[1]$. Then it is clear that $\boldsymbol{Q}(c)=\boldsymbol{Q}\left(B / A^{2}, C / A^{3}\right)$. If $n=6$, by the same argument, the classical result [1] shows that $\boldsymbol{Q}(c)$ is a purely transcendental extension of $\boldsymbol{Q}$ of dimension 3 (cf. also [2]).

In the next place, suppose that $m$ does not divide $n$. Choosing a suitable transformation of the type (II) of Prop. 4, we can transform $Y$ to the curve $Y^{\prime}: y^{m}=x^{n}+x^{n-2}+\sum_{i=0}^{n-3} p_{i} x^{n-3-i}$. Suppose that the $p_{i}$ are algebraically independent over $\boldsymbol{Q}$. Then, by Prop. 4, we see that $\boldsymbol{Q}\left(\zeta, p_{0}^{2}, p_{1}, p_{2}^{2}, p_{3}, \cdots\right)$ is the field $k_{0}$ of Prop. 3 .

5. Let us now consider the curve $Y: y^{m}=p(x)$ in the special case $m=n=5$. Then $h=6$, and

$$
y^{-2} d x, y^{-3} d x, x y^{-3} d x, y^{-4} d x, x y^{-4} d x, x^{2} y^{-4} d x
$$

form a basis of the vector space of differential forms of the first kind. Let $(J, \mathcal{C}, \theta)$ be as in $\S 2$. Define an isomorphism $\theta^{\prime}$ of $\boldsymbol{Q}(\zeta)$ into $\operatorname{End}_{\boldsymbol{Q}}(J)$ so that $\theta^{\prime}(\zeta)=\theta\left(\zeta^{3}\right)$. Hereafter we consider $\mathbb{P}^{\prime}=\left(I, \mathcal{C}, \theta^{\prime}\right)$ instead of $\mathbb{P}$. $\mathbb{P}^{\prime}$ is of type $\left\{\boldsymbol{Q}(\zeta), \Phi^{\prime}, \rho\right\}$, for a representation $\Phi^{\prime}$ such that $\Phi^{\prime}(\zeta)$ is the diagonal matrix with diagonal elements $\zeta, \zeta, \zeta^{-1}, \zeta^{3}, \zeta^{3}, \zeta^{3}$. It is easy to verify that $P$ and $P^{\prime}$ have the same field of moduli. Let $K=\boldsymbol{Q}(\zeta)$, $\zeta=e^{2 \pi i / 5}$, and let $\sigma_{\nu}$, for $\nu=1,2$, be the automorphism of $K$ such that $\zeta^{\sigma}{ }_{\nu}=\zeta^{\nu}$. With the notation $r_{\nu}$ and $s_{\nu}$ of $\S 1$, we have $r_{1}=2, s_{1}=1, r_{2}=0$, $s_{2}=3$. Define an anti-hermitian form $T$ and a lattice $L$ in $K^{3}$ as in $\S 1$, for the present $Q^{\prime}$. The family $\Sigma\left(K, \Phi^{\prime}, \rho ; T, L\right)$ is parametrized by the point in a domain

$$
H=\left\{\left.(z, w) \in \boldsymbol{C}^{2}|| \boldsymbol{z}\right|^{2}+|w|^{2}<1\right\} .
$$

In view of (1.3), $\sqrt{-1} T^{\sigma_{2}}$ is positive definite. Hence $H / \Gamma(T, L)$ is compact.

Now take $p(x)=\sum_{i=0}^{5} p_{i} x^{i}$ so that the $p_{i}$ are algebraically independent over $\boldsymbol{Q}$. Let $k_{0}$ be the field of moduli of $\boldsymbol{P}^{\prime}$. By $[8,1.7], k_{0}$ contains $K=\boldsymbol{Q}(\zeta)$. The consideration of $\S 4$ shows that $k_{0}$ is a purely transcendental extension of $\boldsymbol{Q}(\zeta)$ of dimension 2. By Prop. 1, $\boldsymbol{Q}\left(f_{1}, \cdots, f_{\kappa}\right)$ is a purely transcendental extension of $\boldsymbol{Q}(\zeta)$ of dimension 2 .

6. Our next task is to determine $T$ and $L$ explicitly. Let $C^{h} / D$ and $E$ be as in $\S 1$. In our case of $P^{\prime}=\left(J, \mathcal{C}, \theta^{\prime}\right)$, it is essential that $J$ is a jacobian variety. Since every jacobian variety is self-dual, we have

so that by (1.2),

$$
D=\left\{x \in \boldsymbol{C}^{h} \mid E(x, D) \subset \boldsymbol{Z}\right\},
$$




$$
L=\left\{a \in K^{3} \mid \operatorname{Tr}_{K / Q}(T(a, L)) \subset Z\right\} .
$$

Put $\eta=\zeta^{2}-\zeta^{-2}, S=\eta^{-1} T, \mathfrak{r}=Z[\zeta]$. We see that $\theta(\mathfrak{r}) \subset$ End $(J)$, and hence $L$ is an $\mathfrak{r}$-lattice in $K^{3}$. Since $\eta^{3} \mathfrak{x}$ is the different of $K$ with respect to $\boldsymbol{Q}$, and since $\eta^{4} \mathfrak{r}=5 \mathfrak{r}$, we have

$$
L=\left\{a \in K^{3} \mid S(a, L) \subset 5^{-1} \mathfrak{x}\right\} .
$$

From this relation we can derive the structure of $S$ and $L$ as follows. Let $\left\{e_{1}, e_{2}, e_{3}\right\}$ be a basis of $K^{3}$, and $S_{0}$ a hermitian form on $K^{3}$ represented by the diagonal matrix with diagonal elements $1,1,(1-\sqrt{5}) / 2$ with respect to $\left\{e_{i}\right\}$. Then $S_{0}$ and $S$ have the same signature at every infinite place of $\boldsymbol{Q}(\sqrt{5})$. Let $\mathfrak{a}=5^{-1 / 2} \mathfrak{r}, L_{0}=\mathfrak{a} e_{1}+\mathfrak{a} e_{2}+\mathfrak{a} e_{3}$. Then $L_{0}$ is an r-lattice in $K^{3}$, and we have

$$
L_{0}=\left\{a \in K^{3} \mid S_{0}\left(a, L_{0}\right) \subset 5^{-1} \mathfrak{r}\right\} .
$$

By Prop. 8 of Appendix, there exists a $K$-linear automorphism $\tau$ of $K^{3}$ such that $S_{0}(x \tau, y \tau)=S(x, y)$. Therefore we may put $S=S_{0}$ without loss of generality. By Prop. 6 of Appendix, $L$ and $L_{0}$ are $\mu_{0}$-maximal $\mathfrak{r}$ lattices and $\mu_{0}(L)=\mu_{0}\left(L_{0}\right)=5^{-1} \mathrm{r}$. By Prop. 5 of Appendix, $L$ and $L_{0}$ belong to the same genus with respect to $U\left(S_{0}\right)$. Now $\boldsymbol{Q}(\zeta)$ has the class number 1. Hence by $[7,5.24,(\mathrm{i})]$, there exists an element $\alpha$ of $U\left(S_{0}\right)$ such that $L_{0} \alpha=L$. Therefore taking a suitable coordinate system, we may identify $\Gamma(T, L)$ with the group

$$
\left\{\tau \in G L\left(K^{3}\right) \mid S_{0}(x \tau, y \tau)=S_{0}(x, y), L_{0} \tau=L_{0}\right\} .
$$

Combining this and the result of $\S 5$, we get the assertion of our main theorem in the case (5).

7. We can treat the remaining cases by the same procedure, except the case (3). Let $Y$ be the curve defined by $y^{4}=p(x) q(x)^{2}$, where $p$ and $q$ are polynomials without multiple $\operatorname{root}$, and $\operatorname{deg}(p)=2, \operatorname{deg}(q)=3$; we assume that $p$ and $q$ have no common root. The genus of $Y$ is 3 , and $y^{-1} d x, q(x) y^{-3} d x, x q(x) y^{-3} d x$ form a basis of differential forms of the first kind. As in $\S 2$, from this $Y$ we get $\mathcal{P}=(J, \mathcal{C}, \theta)$ of type $\{Q(i), \Phi, \rho\}$, where $\Phi^{\prime}(i)$ is the diagonal natrix with diagonal elements $i, i,-i$. Define $T$ and $L$ as in $\S 1$. Then $\Sigma(\boldsymbol{Q}(i), \Phi, \rho ; T, L)$ is again parametrized by $H$ of (5.1). Let $p(x)=\sum_{\lambda=0}^{2} p_{\lambda} x^{\lambda}, q(x)=\sum_{\mu=0}^{3} q_{\mu} x^{\mu}$, and let $k_{0}$ be the field of moduli of $P$. Suppose that the $p_{\lambda}$ and $q_{\mu}$ are algebraically independent 
over $\boldsymbol{Q}$. Then we see easily that $\boldsymbol{Q}(i) \subset k_{0} \subset \boldsymbol{Q}\left(i, p_{\lambda}, q_{\mu}\right), \operatorname{dim}_{\boldsymbol{Q}} k_{0}=2$. By virtue of Castelnuovo's theorem (cf. [9]), this, together with Prop. 1, shows that the field of automorphic functions on $H$ with respect to $\Gamma(T, L)$ is purely transcendental over $\boldsymbol{C}$.

To determine $T$ anp $L$, we employ the same argument as in $\S 6$. In this case, 2 is the only prime ramified in $\boldsymbol{Q}(i)$. Therefore, the present situation is somewhat different from $\S 6$. But the consideration in the last part of Appendix is sufficient to determine $T$ and $L$ explicitly from the relation similar to (6.1). Thus we get the whole result of our theorem.

\section{Appendix}

Let $F$ be an algebraic number field of finite degree, and $K$ a quadratic extension of $F$. We denote by $g$ and $\mathfrak{r}$ the ring of integers in $F$ and in $K$ respectively, and by $\rho$ the non-trivial automorphism of $K$ over $F$. Let $V$ be a vector space over $K$ of dimension $n$, and $S(x, y)$ a nondegenerate hermitian form : $V \times V \rightarrow K$, with respect to $\rho$. For every r-lattice $L$ in $V$, we denote by $\mu^{\prime}(L)$ (resp. $\left.\mu_{0}(L)\right)$ the ideal in $F$ (resp. $K$ ) generated by the elements $S(x, x)$ (resp. $S(x, y)$ ) for all $x \in L$ (resp. $x \in L$, $y \in L) . \quad L$ is called maximal (resp. $\mu_{0}$-maximal) if there is no $\mathfrak{r}$-lattice $M$ in $V$, other than $L$, such that $L \subset M$ and $\left.\left.\mu_{0}^{\prime} L\right)=\mu_{0}^{\prime} M\right)\left(\operatorname{resp} . \mu_{0}(L)=\mu_{0}(M)\right)$. For every prime ideal $\mathfrak{p}$ of $F$, let $\mathfrak{g}_{\mathfrak{p}}$ and $F_{\mathfrak{p}}$ denote the completions of $\mathfrak{g}$ and $F$ with respect to $\mathfrak{p}$. Then we put $K_{\mathfrak{p}}=K \otimes_{F} F_{\mathfrak{p}}, \mathfrak{x}_{\mathfrak{p}}=\mathfrak{x}_{\mathfrak{p}}$, $V_{\mathfrak{p}}=V \otimes_{F} F_{\mathfrak{p}} ; \rho$ and $S$ can be extended naturally to $K_{\mathfrak{p}}$ and $V_{\mathfrak{p}}$. We can define similarly $\mu, \mu_{0}$, the maximality, and the $\mu_{0}$-maximality for $\mathfrak{r}_{\mathfrak{p}}$ lattices in $V_{\mathfrak{p}}$. In [7] we investigated maximal lattices. Here we supply some results on $\mu_{0}$-maximal lattices which are necessary for the proof of our theorem.

Let $\delta$ be the different of $K$ with respect to $F$. By $[7,2.11]$, for every $\mathfrak{r}$-lattice $L$ in $V$, we have

$$
\begin{gathered}
\mu(L) \mathfrak{x} \subset \mu_{0}(L) \subset \mu^{\prime}(L) \mathfrak{D}^{-1}, \\
\operatorname{Tr}_{K / F}\left(\mu_{0}(L)\right) \subset \mu(L) .
\end{gathered}
$$

Therefore, if $\mathfrak{p}$ is unramified in $K$, we have $\mu_{0}(L)_{\mathfrak{p}}=\mu(L) \mathfrak{x}_{\mathfrak{p}}$, and hence there is no distinction between maximality and $\mu_{0}$-maximality for the $\mathfrak{x}_{\mathfrak{p}}$-lattices in $V_{\mathfrak{p}}$. If $V$ is one-dimensional, it is clear that every $\mathfrak{r}$-lattice $L$ is maximal and $\mu_{0}$-maximal, and $\mu_{0}(L)=\mu^{\prime}(L) \mathfrak{x}$.

Proposition 1. Let $L$ be a $\mu_{0}$-maximal $\mathfrak{x}_{\mathfrak{p}}$-lattice in $V_{\mathfrak{p}}$ such that $\mu_{0}(L)=\mu(L) \mathcal{D}_{\mathfrak{p}}^{-1}$. Then $L$ is maximal. 
Let $M$ be an $\mathfrak{x}_{\mathfrak{p}}$-lattice such that $L \subset M$ and $\mu(M)=\mu_{0}(L)$. Then $\mu_{0}(L) \subset \mu_{0}(M) \subset \mu(M) \mathfrak{D}_{\mathfrak{p}}^{-1}=\mu_{0}(L) \mathfrak{D}_{\mathfrak{p}}^{-1}=\mu_{0}(L)$, so that $\mu_{0}(L)=\mu_{0}(M)$. Since $L$ is $\mu_{0}$-maximal, we get $L=M$; this shows that $L$ is maximal.

Proposition 2. If $\mathfrak{p}$ does not divide 2 , every maximal $\mathfrak{x}_{\mathfrak{p}}$-lattice in $V_{\mathfrak{p}}$ is $\mu_{0}$-maximal.

By our assumption, for every ideal $\mathfrak{a}_{\mathfrak{p}}$ in $K_{\mathfrak{p}}$, we have

$$
\operatorname{Tr}_{K \mathfrak{p} / F_{\mathfrak{p}}}\left(\mathfrak{a}_{\mathfrak{p}}\right)=\mathfrak{a}_{\mathfrak{p}} \cap F_{\mathfrak{p}} .
$$

Hence, from (A.1) and (A.2), we obtain, for every $\mathfrak{r}_{\mathfrak{p}}$-lattice $L$ in $V_{\mathfrak{p}}$,

$$
\operatorname{Tr}_{K \mathfrak{p} / F_{\mathfrak{p}}}\left(\mu_{0}(L)\right)=\mu_{0}(L) \cap F_{\mathfrak{p}}=\mu^{\prime}(L) .
$$

Then our assertion is obvious.

Proposition 3. Suppose that $n=2, \mathfrak{p}$ does not divide 2 , and $S$ is anisotropic in $V_{\mathfrak{p}}$. Then every $\mu_{0}$-maximal $\mathfrak{r}_{\mathfrak{p}}$-lattice in $V_{\mathfrak{p}}$ is maximal.

If $\mathfrak{p}$ is unramified in $K$, there is no problem; so we assume that $\mathfrak{p}$ is ramified in $K$. Let $L$ be a $\mu_{0}$-maximal $\mathfrak{r}_{\mathfrak{p}}$-lattice in $V_{\mathfrak{p}}$. Since $\mathfrak{p}$ does not divide 2, the relation (A. 1$)$ implies that $\left.\mu_{0}(L)=\mu_{0}^{\prime} L\right) \mathfrak{r}_{\mathfrak{p}}$ or $\mu_{0}(L)$ $=\mu(L) \mathfrak{D}_{\bar{p}}^{-1}$. If $\left.\mu_{0}(L)=\mu^{\prime} L\right) \mathfrak{d}_{\bar{p}}^{-1}, L$ is maximal by virtue of Prop. 1 . Assume that $\mu_{0}(L)=\mu_{(}(L) \mathfrak{r}_{p}$. Then there exists an element $x$ of $L$ such that $\mu_{0}(L)=(S(x, x))$. Put $L^{\prime}=\{y \in L \mid S(x, y)=0\}$. We can easily verify that $L=\mathfrak{r}_{\mathfrak{p}} x+L^{\prime}$. Since $V$ is two-dimensional, we have $L^{\prime}=\mathfrak{x}_{\mathfrak{p}} y$ for some $y$. Since $L$ is $\mu_{0}$-maximal and $\mathfrak{p}$ is ramified in $K$, we must have $(S(y, y))$ $=\mu_{0}(L)$. Now put $\left.M=\left\{u \in V \mid S(u, u) \in \mu^{\prime} L\right)\right\}$. By [7,4.5], $M$ is a maximal $\mathfrak{x}_{\mathfrak{p}}$-lattice in $V_{\mathfrak{p}}$. We have clearly $L \subset M$. Let $u=a x+b y \in M$ with $a, b$ in $K_{\mathfrak{p}}$. Then

$$
a a^{\rho} S(x, x)+b b^{\rho} S(y, y) \in \mu(L)=(S(x, x)) .
$$

Put $c=S(x, x)^{-1} S(y, y)$. Then $c$ is a unit in $\mathfrak{g}_{\mathfrak{p}}$, and $a a^{p}+b b^{p} c \in \mathfrak{g}_{p}$. Let $\pi$ be a prime element of $\mathfrak{x}_{\mathfrak{p}}$. Assume that $u \notin L$. Then $\pi^{e} a$ and $\pi^{e} b$ are units in $\mathfrak{x}_{\mathfrak{p}}$ with a positive integer $e$, and

$$
\left(\pi^{e} a\right)\left(\pi^{e} a\right)^{\rho}+\left(\pi^{e} b\right)\left(\pi^{e} b\right)^{\rho} c \equiv 0 \quad \bmod \left(\pi \pi^{\rho}\right)^{e} \mathfrak{g}_{p} .
$$

It follows that $-c$ is the norm of an element in $K_{\mathfrak{p}}$. But this is a contradiction, since $S$ is anisotropic in $V_{\mathfrak{p}}$. Therefore $u \in L$, and hence $M=L$. This proves our proposition.

Proposition 4. Suppose that $\mathfrak{p}$ does not decompose in $K$. When $\mathfrak{p}$ divides 2, suppose further that $\mathfrak{p}$ is unramified in $K$. Let $L$ be a $\mu_{0}-$ 
maximal $\mathfrak{x}_{\mathfrak{p}}$-lattice in $V_{\mathfrak{p}}$. Put $\mathfrak{b}=\mu_{0}(L)$. Then there exists a Witt decomposition $\quad V_{\mathfrak{p}}=\sum_{i=1}^{m}\left(K_{\mathfrak{p}} x_{i}+K_{\mathfrak{p}} y_{i}\right)+W \quad$ (cf. [7,4.3]) such that $L=\sum_{i=1}^{m}\left(\mathfrak{x}_{\mathfrak{p}} x_{i}+\mathfrak{b} y_{i}\right)+M$, where $M$ is a maximal $\mathfrak{x}_{\mathfrak{p}}$-lattice in $W$ given by $M=\{z \in W \mid S(z, z) \in \mu(L)\}$. Conversely, let $\mathfrak{b}$ be an ideal in $K_{\mathfrak{p}}$, and $V_{\mathfrak{p}}=\sum_{i=1}^{m}\left(K_{\mathfrak{p}} x_{i}+K_{\mathfrak{p}} y_{i}\right)+W$ be a Witt decomposition. Let

$$
M=\left\{z \in W \mid S(z, z) \in \mathfrak{b} \cap F_{\mathfrak{p}}\right\}, \quad L=\sum_{i=1}^{m}\left(\mathfrak{x}_{\mathfrak{p}} x_{i}+\mathfrak{b} y_{i}\right)+M
$$

Then $L$ is a $\mu_{0}$-maximal $\mathfrak{x}_{\mathfrak{p}}$-lattice in $V_{\mathfrak{p}}$.

The converse part can be proved in a straightforward way. The proof of the direct part is similar to the proof of $[7,4.7]$; so here we only sketch a proof. Assume that $S$ is isotropic in $V_{\mathfrak{p}}$. Then we can find an element $x$ in $V_{\mathfrak{p}}$ such that $S(x, x)=0$ and $\mathfrak{x}_{\mathfrak{p}}=\left\{a \in K_{\mathfrak{p}} \mid a x \in L\right\}$. Put $\mathfrak{a}=S(x, L)$. We get easily $\mu_{0}\left(\mathfrak{a}^{-1} \mathfrak{b} x+L\right)=\mu_{0}(L)$, so that $\mathfrak{a}^{-1} \mathfrak{b} x+L=L$ by virtue of the $\mu_{0}$-maximality of $L$. We have therefore $L \supset \mathfrak{a}^{-1} \mathfrak{b} x$, and hence $\mathfrak{a}^{-1} \mathfrak{b} \subset \mathfrak{x}_{\mathfrak{p}}$. It follows that $S(x, L)=\mathfrak{b}$. Therefore we find an element $u \in L$ such that $\mathfrak{b}=(S(x, u))$. Our assumption implies $\mu(L)=\operatorname{Tr}_{K \mathfrak{p} / F \mathfrak{p}}(\mathfrak{b})$ $=T r_{K \mathfrak{p} / F \mathfrak{p}}\left(S(x, u) \mathfrak{r}_{\mathfrak{p}}\right)$. Using this fact, we can find an element $\lambda$ of $\mathfrak{x}_{\mathfrak{p}}$ such that $S(x+\lambda u, x+\lambda u)=0$. Put $y=x+\lambda u, L^{\prime}=\{z \in L \mid S(x, z)=S(y, z)=0\}$. Then we have $L=\mathfrak{x}_{\mathfrak{p}} x+\mathfrak{b} y+L^{\prime}$. Applying induction to $L^{\prime}$, we get our assertion, in view of Prop. 3.

Let $U(S)$ be the group of all $K$-linear automorphisms $\sigma$ of $V$ such that $S(x \sigma, y \sigma)=S(x, y)$. As in $[7,5.18]$ we define genera of $\mathfrak{r}$-lattices in $V$.

Proposition 5. Suppose that every prime factor of 2 in $F$ is unramified in $K$. Let $L$ be a $\mu_{0}$-maximal $\mathfrak{x}$-lattice in $V$. Then the genus of $L$ with respect to $U(S)$ consists of all $\mu_{0}$-maximal $\mathfrak{x}$-lattices $M$ such that $\mu_{0}(M)=\mu_{0}(L)$.

This follows directly from $[7,3.3]$ and Prop. 4 by the same argument as in the proof of $[7,5.25]$.

Proposition 6. Suppose that every prime factor of 2 in $F$ is unramified in $K$. Let a be an ideal in $F$, and $L$ an $\mathfrak{r}$-lattice in $V$. Suppose that $L=\{x \in V \mid S(x, L) \subset \mathfrak{a r}\}$. Then $L$ is $\mu_{0}$-maximal, and $\mu_{0}(L)=\mathfrak{a r}$.

Our assertion is clear if $n=1$. Suppose that $n>1$. For every $\mathfrak{r}-$ lattice $M$ in $V$, define $M^{*}$ by $M^{*}=\{x \in V \mid S(x, M) \subset \mathfrak{a r}\}$. We see that $M \subset M^{*}$ if and only if $\mu_{0}(M) \subset a \mathfrak{a r}$. Since $L=L^{*}$, we have $\mu_{0}(L) \subset a$ r. If $M_{1} \subset M_{2}$, then $M_{1}^{*} \supset M_{2}^{*}$. Now let $L \subset M, \mu_{0}(M) \subset a$ ar. Then we have $M^{*} \subset L^{*}=L \subset M \subset M^{*}$, so that $L=M$. This shows that $L$ is $\mu_{0}$-maximal. 
By $[7,3.2]$ and by Prop. 4 , we can easily find a $\mu_{0}$-maximal $\mathfrak{r}$-lattice $L^{\prime}$ such that $L \subset L^{\prime}$ and $\mu_{0}\left(L^{\prime}\right)=\mathfrak{a r}$. Then the above argument shows again $L=L^{\prime}$. This proves our proposition.

Proposition 7. Let $\mathfrak{p}$ be a prime ideal in $F$ which remains prime in $K$. Suppose that there exist an ideal $\mathfrak{a}$ in $F_{\mathfrak{p}}$ and an $\mathfrak{x}_{\mathfrak{p}}$-lattice $L$ in $V_{\mathfrak{p}}$ such that $L=\left\{x \in V_{\mathfrak{p}} \mid S(x, L) \subset \mathfrak{a r}_{\mathfrak{p}}\right\}$. Then the structure $\left(V_{\mathfrak{p}}, S\right)$ is uniquely determined by $\mathfrak{a}$. More precisely, if $n$ is odd and $\mathfrak{a}=a \mathfrak{g}_{\mathfrak{p}}, d(S)$ is the class of $(-1)^{(n-1) / 2} a$ modulo $N_{K \mathfrak{p} / F_{\mathfrak{p}}}\left(K_{\mathfrak{p}}^{*}\right)$ (cf. $[7,2.1$ and 4.2]). If $n$ is even, $S$ is maximally isotropic in $V_{\mathfrak{p}}$, namely, $V_{\mathfrak{p}}$ has the trivial kernel subspace with respect to $S$ (cf. [7,4.3]).

By Prop. 4, we find a Witt decomposition $V_{\mathfrak{p}}=\sum_{i=1}^{m}\left(K_{\mathfrak{p}} x_{i}+K_{\mathfrak{p}} y_{i}\right)+W$ such that $L=\sum_{i=1}^{m}\left(\mathfrak{x}_{\mathfrak{p}} x_{i}+\mathfrak{b} y_{i}\right)+M, M=\{u \in W \mid S(u, u) \in \mathfrak{a}\}$. By our assumption on $L$, we have

$$
M=\left\{u \in W \mid S(u, M) \subset \mathfrak{a} \mathfrak{r}_{\mathfrak{p}}\right\}
$$

If $n$ is odd, we have $W=K_{\mathfrak{p}} z, W=\mathfrak{x}_{\mathfrak{p}} z$ for some $z$. Hence (A. 5) implies that $\mathfrak{a}=S(z, z) \mathfrak{g}_{\mathfrak{p}}$. Since $\mathfrak{p}$ is unramified in $K$, every unit in $\mathfrak{g}_{\mathfrak{p}}$ is the norm of an element of $K_{\mathfrak{p}}$. Therefore we get our assertion for odd $n$. Next assume that $n$ is even and $W$ is two-dimensional. Since $\mu_{0}(M)$ $=\mu(M) \mathfrak{x}_{\mathfrak{p}}=\mathfrak{a x}_{\mathfrak{p}}$, we find, using the argument of the proof of Prop. 3, an expression $M=\mathfrak{x}_{\mathfrak{p}} u+\mathfrak{x}_{p} v$ with $S(u, v)=0$. On account of (A.5), we see that $\mathfrak{a}=S(u, u) \mathfrak{g}_{\mathfrak{p}}=S(v, v) \mathfrak{g}_{\mathfrak{p}}$; hence $S(u, u)^{-1} S(v, v)$ is a unit in $\mathfrak{g}_{\mathfrak{p}}$. Therefore we find an element $c$ in $K_{\mathfrak{p}}$ such that $c c^{\rho}=-S(u, u)^{-1} S(v, v)$. Then we get $S(c u+v, c u+v)=0$, which is a contradiction. Hence $S$ must be maximally isotropic in $V_{\mathfrak{p}}$.

Proposition 8. Suppose that there is no or only one prime ideal in $F$ which is ramified in $K$. Suppose that there exist an ideal a in $F$ and an $\mathfrak{r}$-lattice $L$ such that $L=\{x \in V \mid S(x, L) \subset a \mathfrak{a r}\}$. Then the structure $(V, S)$ is uniquely determined, up to isomorphism, by a and the signature of $S$ at infinite prime spots of $F$.

Let $\mathfrak{q}$ be a possible prime ideal in $F$ which is ramified in $K$. By Prop. 7 , the structure $\left(V_{\mathfrak{p}}, S\right)$ is uniquely determined by $\mathfrak{a}$ if $\mathfrak{p} \neq \mathfrak{q}$. If we assign a fixed signature to each infinite prime spot of $F$, then the structure $\left(V_{\mathrm{q}}, S\right)$ is automatically determined by virtue of the product formula of norm residue symbol. This proves our proposition.

If a prime factor of 2 in $F$ is ramified in $K$, we can not apply Prop. 5. However, under a suitable condition, we may treat such a case. 
For example, let us consider the case where $F=\boldsymbol{Q}, K=\boldsymbol{Q}(i), i^{2}=-1$, $n=3$. Let $\mathfrak{p}=(2), \mathfrak{P}=(1+i)$, and let $L$ be an $\mathfrak{r}_{\mathfrak{p}}$-lattice in $V_{\mathfrak{p}}$ such that

$$
L=\left\{x \mid S(x, L) \subset\left(2^{-1}\right)\right\} \text {. }
$$

Assume that $S$ is represented in $V_{\mathfrak{p}}$ by the diagonal matrix with diagonal elements $1,1,-1$. Now by $[7,4.15]$, the following two cases may occur.

(I) $L=\mathfrak{r}_{\mathfrak{p}} x+\mathfrak{r}_{\mathfrak{p}} y+\mathfrak{r}_{\mathfrak{p}} z, S(x, y)=S(y, z)=S(z, x)=0$.

(II) $L=\mathfrak{x}_{\mathfrak{p}} x+\mathfrak{x}_{\mathfrak{p}} y+\mathfrak{x}_{\mathfrak{p}} z, S(x, z)=S(y, z)=0$,

$$
S(x, x) \in \mathfrak{P S}(x, y), S(y, y) \in \mathfrak{P S}(x, y) \text {. }
$$

Put $S(x, x)=a, S(y, y)=b, S(z, z)=c, S(x, y)=d$. In the case (I), by (A. 6), we have $(a)=(b)=(c)=\left(2^{-1}\right)$. Put $2 a=a^{\prime}, 2 b=b^{\prime}, 2 c=c^{\prime}$. By our assumption on $S,-a^{\prime} b^{\prime} c^{\prime}$ must be the norm of an element of $K_{\mathfrak{p}}$ (cf. [7, 4.2]). Since -1 is not a norm residue, we may assume, exchanging the order of $x, y, z$ if necessary, that $a^{\prime}=b^{\prime}=c^{\prime}=-1$ or $a^{\prime}=b^{\prime}=1, c^{\prime}=-1$. The former case can be reduced to the latter case by the transformation $u=e^{-1}(x-(1+i) y), v=e^{-1}((1-i) x+y), w=z$, where $e$ is an element of $\mathfrak{x}_{\mathfrak{p}}$ such that $e e^{\rho}=-3$.

In the case (II), by (A.6), we have $(c)=(d)=\left(2^{-1}\right)$, so that $a \in \mathfrak{g}_{\mathfrak{p}}$, $h \in a_{\mathfrak{p}}$. Hence $d d^{\mathfrak{p}}-a b$ is the norm of an elemet of $K_{\mathfrak{p}}$. Therefore, by $[7,4.1], S$ is isotropic in $K_{\mathfrak{p}} x+K_{\mathfrak{p}} y$. It follows that $c$ is the norm of an element of $K_{\mathfrak{p}}$, on account of our assumption on $S$. Hence we may assume $\mathfrak{x}_{\mathfrak{p}} z=\mathfrak{B}^{-1} w$ with $S(w, w)=1$. Put $M=\mathfrak{x}_{\mathfrak{p}} x+\mathfrak{x}_{\mathfrak{p}} y$. Then $\mu_{0}(M) \subset \mathfrak{g}_{\mathfrak{p}}$ $\left.=2 \mu_{0}(M) \subset \mu^{\prime} M\right)$, so that $2 \mu_{0}(M)=\mu^{\prime}(M)$. Applying the argument of the proof of Prop. 6 to $M$, we see that $M$ is $\mu_{0}$-maximal, so that by Prop. 1, $M$ is maximal. By $[7,4.7]$, we have $M=\mathfrak{F}^{-1} u+\mathfrak{\beta}^{-1} v$ with $S(u, u)=S(v, v)$ $=0, S(u, v)=1$. Put $r=u+w, s=v-w, t=u-v+w$. Then $S(r, r)=S(s, s)$ $=1, S(t, t)=-1, S(r, s)=S(s, t)=S(t, r)=0, \quad L=\mathfrak{P}^{-1} r+\mathfrak{P}^{-1} s+\mathfrak{P}^{-1} t$. Therefore $L$ is reduced to the case (I).

This result, combined with Prop. 4 and a localization of Prop. 6, shows that every $\mathfrak{x}$-lattice $L$ in $V$ satisfying (A.6) belongs to one and the same genus with respect to $U(S)$.

Osaka University and Princeton University

\section{References}

[1] A. Clebsch: Theorie der binären algebraischen Formen, Leipzig, 1872.

[2] J. Igusa: Arithmetic variety of moduli for genus two, Ann. of Math. 72 (1960), 612-649. 
[3] E. Picard: Sur des fonctions de deux variables indépendantes analogues aux fonctions modulaires, Acta Mathematica 2 (1883), 114-135.

[4] G. Shimura: On the theory of automorphic functions, Ann. of Math. 70 (1959), 101-144.

[5] - : On the zeta-functions of the algebraic curves uniformized by certain automorphic functions, J. Math. Soc. Japan 13 (1961), 275-331.

[6] - On analytic families of polarized abelian varieties and automorphic functions, Ann. of Math. 78 (1963), 149-192.

[7] - Arithmetic of unitary groups, Ann. of Math. 79 (1964), 369-409.

[8] - On the field of definition for a field of automorphic functions, Ann. of Math. 80 (1964), 160-189.

[9] O. Zariski: On Castelnuovo's criterion of rationality $p_{a}=P_{2}=O$ of an algebraic surface, Illinois J. Math. 2 (1958), 303-315. 